Farum

Sociológico

\section{Forum Sociológico}

Série II

24 | 2014

Circulação de saberes e desafios em saúde

\title{
La Investigación Cualitativa en el ámbito de los Servicios de Salud en España: caminos recorridos y reflexiones desde el presente
}

Qualitative research in the field of Health Services in Spain: Progress and current reflections

\section{Carlos Calderón}

\section{OpenEdition}

Journals

Edición electrónica

URL: https://journals.openedition.org/sociologico/1030

DOI: 10.4000/sociologico.1030

ISSN: 2182-7427

Editor

CICS.NOVA - Centro Interdisciplinar de Ciências Sociais da Universidade Nova de Lisboa

Edición impresa

Paginación: 39-45

ISSN: 0872-8380

\section{Referencia electrónica}

Carlos Calderón, «La Investigación Cualitativa en el ámbito de los Servicios de Salud en España: caminos recorridos y reflexiones desde el presente», Forum Sociológico [En línea], 24 | 2014, Puesto en línea el 01 noviembre 2014, consultado el 30 marzo 2022. URL: http://journals.openedition.org/ sociologico/1030 ; DOI: https://doi.org/10.4000/sociologico.1030

Este documento fue generado automáticamente el 30 marzo 2022.

(c) CICS.NOVA 


\title{
La Investigación Cualitativa en el ámbito de los Servicios de Salud en España: caminos recorridos y reflexiones desde el presente ${ }^{1}$
}

\author{
Qualitative research in the field of Health Services in Spain: Progress and \\ current reflections
}

Carlos Calderón

\section{Introducción}

1 Mediante la presente ponencia pretendo compartir una serie de reflexiones acerca de la evolución de la Investigación Cualitativa (IC) en el ámbito de los servicios de salud en España. Aunque con retraso respecto de lo acontecido en el medio anglosajón (Paley \& Lilford, 2011) la publicación de trabajos cualitativos en el campo de la salud se ha incrementado notablemente, comprendiendo una amplia variedad de áreas temáticas y compartiendo asimismo determinadas inquietudes y problemas referidos principalmente a la coherencia metodológica y a la calidad de las publicaciones (Mira et al., 2004; Calderón, 2004; Pope \& Mays, 2009; Pujol et al., 2011).

2 En este caso, tomaré como referencia los resultados de una revisión llevada a cabo acerca de la producción cualitativa publicada e indexada en el Índice Médico Español (IME) en los últimos 30 años, e intentaré extraer algunas conclusiones que nos puedan servir de guión de debate sobre los retos futuros en este campo.

Como se podrá comprobar, se trata de una visión aproximativa, expresamente limitada a la IC en los servicios de salud en España, dado que es éste el ámbito en el que trabajo como Médico de Familia en un Centro de Atención Primaria del Servicio Vasco de Salud, y sin duda condicionada por mis propias experiencias docentes e investigadoras en IC durante las dos últimas décadas. No obstante, confío en que las siguientes 
consideraciones puedan ser también de utilidad para los profesionales de Portugal y de otros países de nuestro contexto iberoamericano.

\section{Material y métodos}

4 Tal y como se avanzó previamente, el presente trabajo se basa en la revisión de la producción cualitativa publicada e indexada en el Índice Médico Español (IME) desde 1982 a 2011. Como es sabido, el IME constituye la mayor base de datos bibliográfica sobre publicaciones de ciencias de la salud en español. Producida por el Consejo Superior de Investigaciones Científicas (CSIC), contiene referencias bibliográficas de artículos de revistas médicas españolas de carácter científico (básicas, experimentales y clínicas), incluyendo todas las áreas de las ciencias de la salud (Medicina, Enfermería, Odontología) en cualquier soporte (impreso o electrónico) desde 1971.

5 En la presente búsqueda se utilizaron los siguientes descriptores: investigación cualitativa, grupos focales, entrevistas en profundidad, estudio etnográfico, análisis cualitativo, teoría fundamentada, análisis temático y análisis del discurso, procediéndose posteriormente a la depuración de aquellos artículos que no se correspondían con el objeto de la revisión.

6 Para el análisis de los resultados, los 291 artículos finalmente seleccionados se distribuyeron por años de publicación, ámbitos de origen y áreas temáticas.

\section{Resultados}

\section{• ¿Creciendo? ¿Para qué audiencias?}

7 En primer lugar, y desde el punto de vista de la cuantificación de la producción investigadora publicada, es importante subrayar el notable incremento que tuvo lugar a partir de mediados de los años 90, principalmente en revistas de Enfermería, Atención Primaria y Salud Pública-Gestión Sanitaria. Si nos atenemos a la información extraída y analizada, se confirma dicha evolución (Figura 1), con un pico máximo en 2005 y un trazado más irregular, e incluso de cierto estancamiento en los últimos años, si bien habría que tener en cuenta asimismo el número posiblemente también creciente aunque escaso - de trabajos publicados en revistas no españolas.

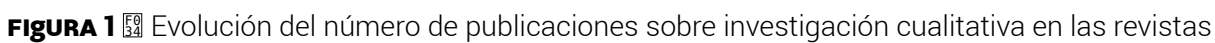
indexadas en el IME

Image 2000001300003CDF00001EB5309252CF.wmf

8 En este mismo sentido, es importante constatar la existencia durante los últimos 10 años de un número cada vez mayor de tesis doctorales cualitativas, de proyectos financiados por agencias autonómicas o estatales, y de actividades docentes tanto en la formación de grado como de postgrado, sobre todo en el campo de la enfermería.

9 Sin embargo, y a pesar de dicho incremento, el número de publicaciones indexadas continúa siendo muy minoritario en comparación con el resto de la producción investigadora, y todavía proporcionalmente menor en las revistas sanitarias de otras especialidades distintas de la Medicina de Familia, Enfermería y Salud Pública. Téngase en cuenta que sólo desde el año 2000 el número de artículos publicados en las 499 
revistas actualmente indexadas en el IME fue de más de 77000 , mientras que en nuestro caso no llegamos a los 270 artículos en no más de 25 revistas.

En mi opinión, las carencias en la producción de trabajos de IC en el ámbito de los servicios de salud no son sólo de número. Por un lado, desconocemos el impacto de lo publicado en la mejora de los problemas que se investigan, pero me temo que, de forma similar a lo que ocurre con la cada vez mayor cantidad de publicaciones de salud en general (Merenstein et al., 2003), también aquí existen importantes vacíos. La pluralidad de corrientes y enfoques teórico-metodológicos que confluyen en la investigación cualitativa no debe ser excusa para dejar de mejorar la calidad de los trabajos de investigación (Reynolds et al., 2011), como tampoco lo es la inadecuación de los criterios de calidad vigentes en los estudios cuantitativos (Barbour \& Barbour, 2003). La calidad de las investigaciones cualitativas requiere de criterios propios que se correspondan con la pluralidad, flexibilidad y particularidades de sus diseños, y que por tanto nos permitan conocer no sólo la cantidad sino también la calidad de lo que se publica (Calderón, 2009). La evaluación de las investigaciones en general, y de las cualitativas en particular, requiere además ir más allá del ámbito de "lo interno" de la labor investigadora y, por supuesto, de los índices de impacto (Niederkrotenthaler et al., 2011) y de la mayor o menor longitud de los currículos de los autores. Conocer y justificar el por qué, para qué y para quién investigamos y publicamos, y sus repercusiones en la mejora de los problemas de partida, constituye por tanto una tarea a la que deberíamos dar respuesta.

11 Pero además considero preocupante la concentración casi exclusiva de trabajos publicados en revistas de ámbitos muy concretos (Enfermería, Atención Primaria, Salud Pública-Gestión Sanitaria, y en menor medida Salud Mental) (Figura 2).

Figura 2 通 Distribución de los ámbitos de las revistas donde se publican los artículos sobre investigación cualitativa

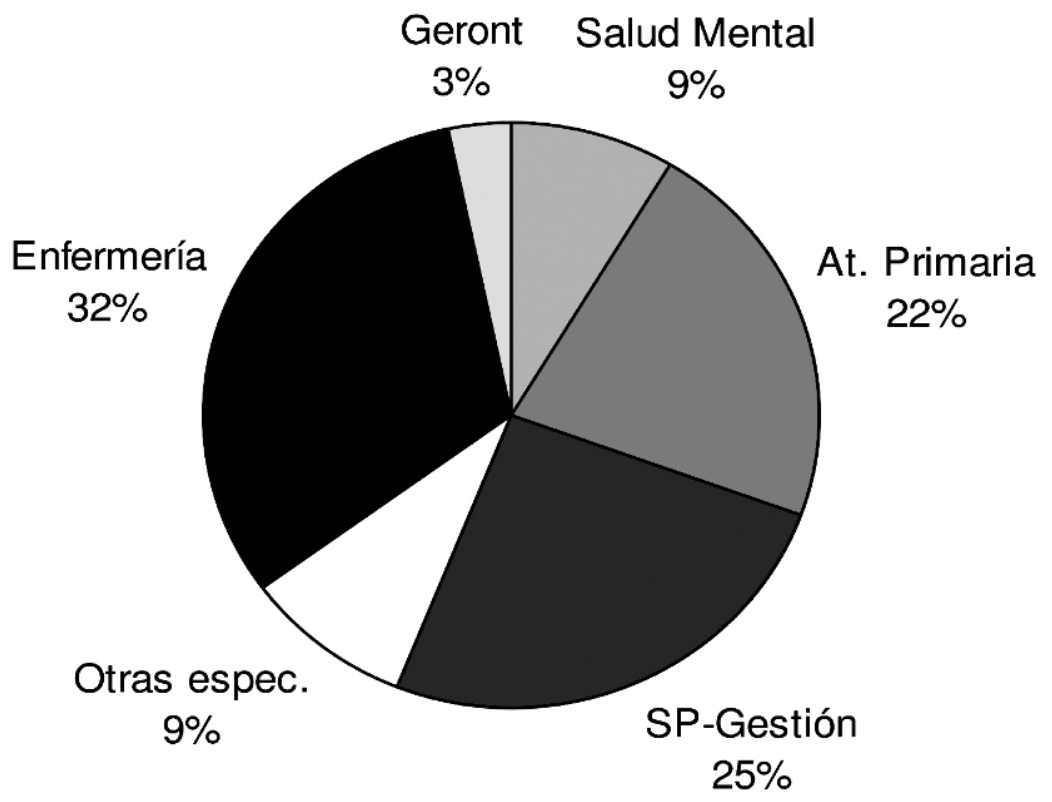

Forum Sociológico, 24 | 2014 
12 Sin duda es explicable, y en cierta medida hemos de sentirnos orgullosos de ello, que seamos las disciplinas más próximas a lo humano donde hayamos desarrollado más la perspectiva holística que representan las metodologías cualitativas. Pero a su vez entiendo que corremos un cierto riesgo de endogamia, al investigar, publicar y difundir la investigación cualitativa en salud casi exclusivamente para nuestras propias audiencias. La perspectiva cualitativa conlleva una apertura y un enriquecimiento potencial de las ciencias de la salud que afecta a todas las especialidades y disciplinas, y por ello deberíamos ser ambiciosos a la hora de difundirla en territorios que no son los nuestros, pero en los que confluyen asimismo vivencias, motivaciones y valores de enfermos y de profesionales, y donde también se explicitan las asimetrías de saberes y poderes de unos y otros en relación a problemas que requieren ser mejor conocidos e interpretados para poderse mejorar en beneficio de los ciudadanos.

\section{• ¿Sobre qué y desde dónde?}

13 En relación con lo anterior, el segundo tema de reflexión tiene que ver con la temática de los trabajos publicados y la procedencia de los autores de las investigaciones.

14 Si tomamos como ejemplo el año 2005, en el que consta el mayor número de publicaciones cualitativas publicadas, resulta difícil delimitar con precisión los objetos de estudio de las publicaciones analizadas ya que son frecuentes las intersecciones entre unos y otros, pero de forma esquemática pueden identificarse ocho áreas temáticas principales: vivencias de los enfermos sobre sus enfermedades, relaciones profesionales-enfermos, hábitos y actividades de promoción y prevención, cuidados, condicionantes del medio social-comunitario, aportaciones teórico-metodológicas, funcionamiento de los servicios, y vivencias de los profesionales sanitarios (Figura 3). 
Figura 3 通 Distribución de las áreas temáticas de las publicaciones sobre investigación cualitativa en 2005

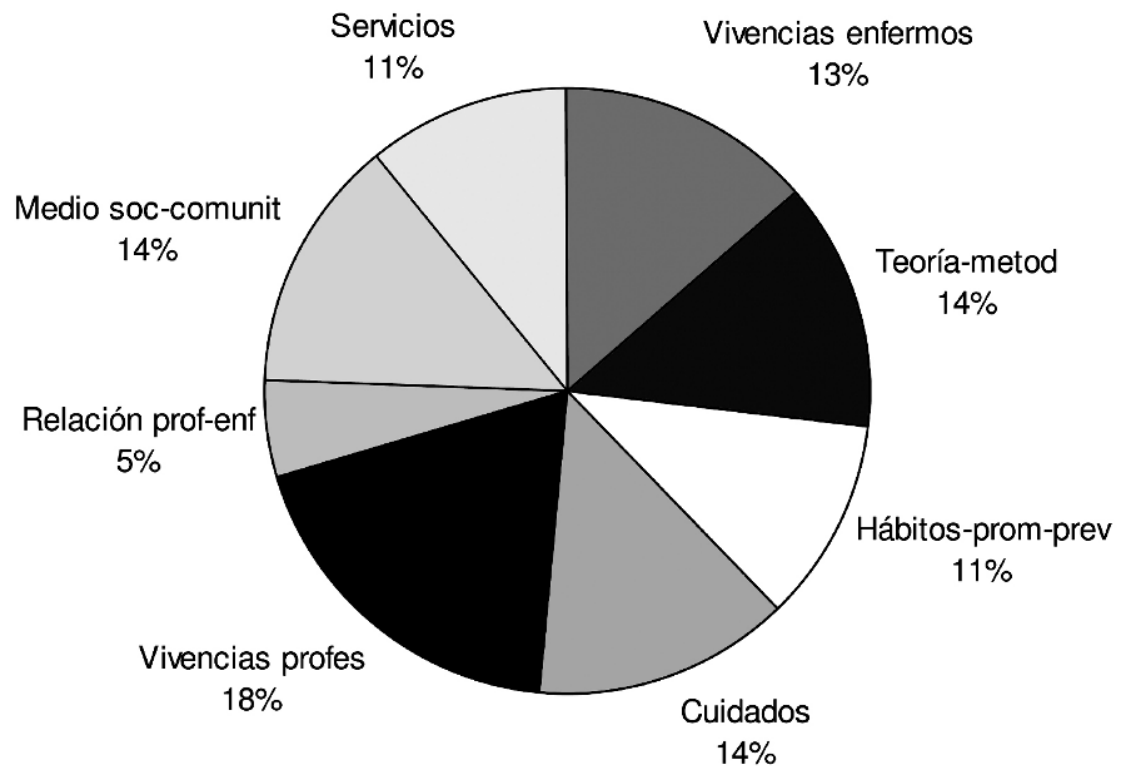

15 En su conjunto, las áreas de distribución de las publicaciones confirman la diversidad de ámbitos de aplicación y el gran potencial de desarrollo de las metodologías cualitativas en el campo de los servicios de salud, pudiendo recomendarse en todo caso la conveniencia de hacer mayores esfuerzos de acercamiento a las complejas realidades de los enfermos, sus cuidadores-as y sus relaciones con los profesionales sanitarios, en cuanto que son aquellos quienes constituyen la justificación principal de dichos servicios.

En este sentido, la información relativa a la procedencia de los autores de las publicaciones resulta ilustrativa. Siguiendo con el año 2005 a modo de ejemplo, comprobamos que el 70 \% se sitúa en Universidades y Escuelas de Enfermería, el $14 \%$ en ámbitos de la gestión y sólo un $13 \%$ en establecimientos clínicos (3 hospitalarios y 2 extra hospitalarios) (Figura 4). 


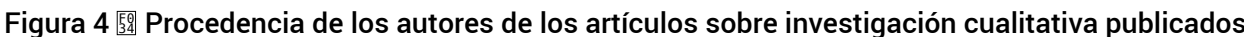
en 2005

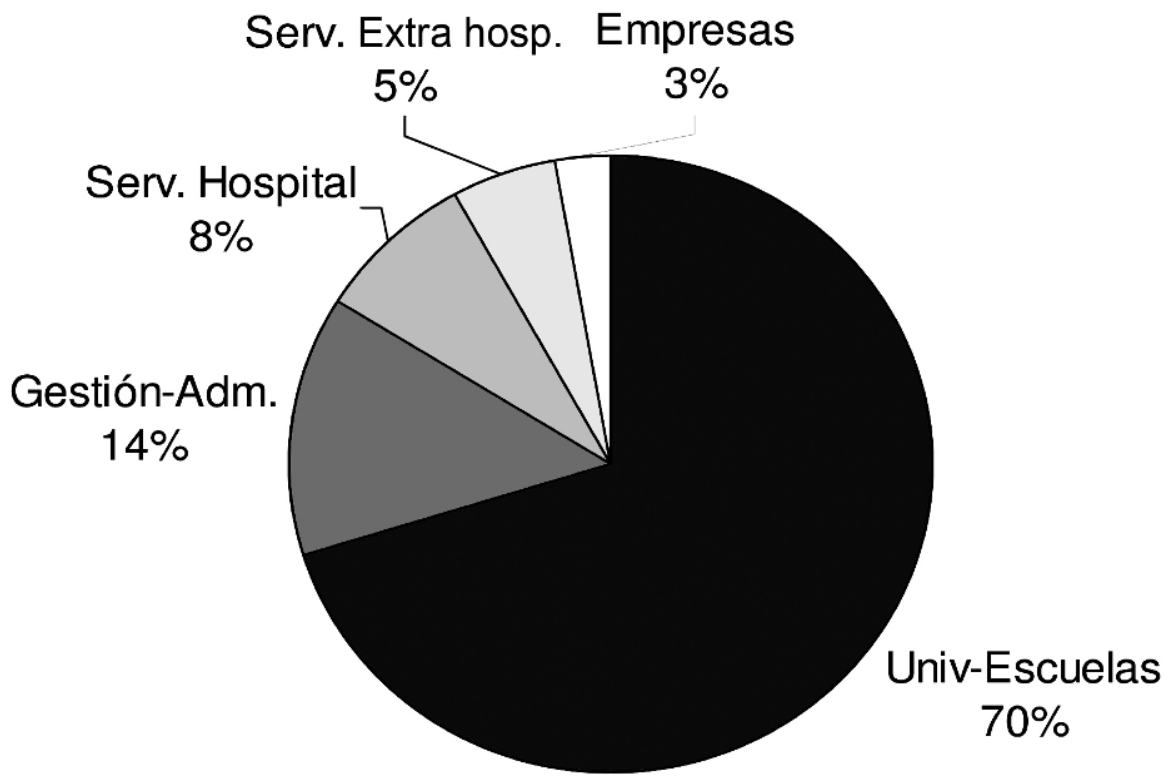

Como comentábamos anteriormente a propósito de las revistas donde se publican los trabajos cualitativos, también aquí es entendible e importante que la academia desempeñe un papel destacado en la producción investigadora, y en algunos casos es cierto que junto a los primeros firmantes también figura algún profesional de otros ámbitos. Pero a la luz de la información expuesta, resulta inevitable apuntar ciertas inquietudes.

Ya no se trataría sólo del riesgo de que la Enfermería produzca para la Enfermería, la Atención Primaria para la Atención Primaria, y la Salud Pública para la Salud Pública, sino que además, la escasa presencia de investigaciones cualitativas lideradas desde los servicios de salud debería alertarnos sobre el peligro de un excesivo encerramiento de la investigación cualitativa en el ámbito académico, y en menor medida en la gestión, lo cual, a su vez, podría contribuir al alejamiento entre la erudición teórica y las preguntas de investigación que surgen de la práctica sanitaria, y quizás explicar en parte la todavía limitada presencia de la investigación cualitativa en los servicios de salud.

\section{• ¿Cómo?}

En tercer lugar, y de forma muy resumida, me he permitido señalar al menos dos tendencias, con sus posibles alternativas, en lo que respecta a los enfoques teóricometodológicos de la producción investigadora (Figura 5). 
Figura 5 [ [0s4 Esquemas de tendencias y propuestas
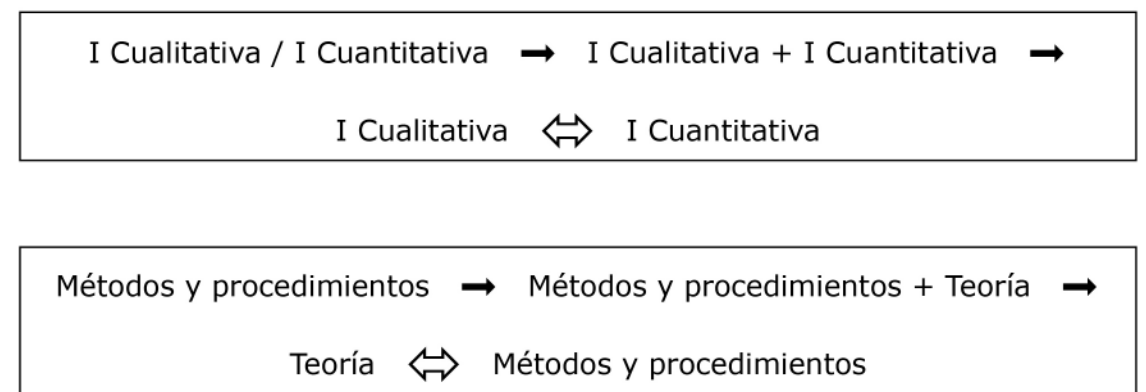

La primera tiene que ver con la relación entre la IC y otras metodologías, principalmente estadístico-epidemiológicas, denominadas como cuantitativas. Durante bastante tiempo la IC se ha presentado, difundido y explicado, en relacióncontraposición a las investigaciones cuantitativas, lo cual sin duda es explicable teniendo en cuenta la todavía hoy predominante identificación en el campo de la salud de la investigación con la cuantificación. Pero a su vez dicho enfoque ha conllevado con frecuencia una excesiva simplificación tanto de la necesaria pluralidad metodológica en el proceso del conocer, como de los referentes teórico-filosóficos de unas y otras metodologías. Cuantificación era igual a positivismo, y la IC debía ir necesariamente acompañada de una etiqueta (principalmente fenomenología, etnografía o teoría fundamentada) sin que los investigadores supieran muy bien el significado y la justificación del etiquetado.

21 En la actualidad se ha avanzado en este sentido, conocemos cada vez mejor los múltiples derroteros de las corrientes de pensamiento que se entrecruzan en el amplio campo de la IC, y en relación a los métodos epidemiológicos y estadísticos asumimos la necesidad de contar con múltiples herramientas para poder conocer e intervenir sobre los problemas y necesidades relacionados con la salud.

No obstante considero que queda bastante camino por recorrer, ya que dicho conocimiento requiere ir más allá de lo sumatorio y desarrollar modos de integración más acordes con las exigencias teóricas y metodológicas derivadas de la complejidad de los objetos de estudio. Se trata de un camino abierto que cuenta ya con una creciente presencia bibliográfica tanto en el diseño de los denominados métodos mixtos (Creswell, 2011;Teddlie \& Tashakkori, 2011), como en lo referente a la síntesis y revisiones sistemáticas de las publicaciones cuantitativas y cualitativas en salud (Pawson et al., 2005), y que sin duda conlleva implicaciones teóricas y epistemológicas acerca del conocimiento científico en salud, por lo que en el marco de esta breve ponencia sólo podemos animar a compartir su debate en profundidad.

$\mathrm{Y}$ en segundo término, entiendo que dichas carencias integradoras se han dado también en el seno de los propios diseños de las investigaciones cualitativas. Por un lado, todavía hoy nos encontramos con investigaciones publicadas en las que la justificación metodológica se reduce, por ejemplo, a la obtención de información mediante grupos focales. Y por otro, también podemos encontrar ejemplos en los que el desarrollo teórico de la adscripción de los autores casi nos permite visualizar desde la introducción cuáles van a ser los resultados del estudio. 

una serie de métodos y técnicas diferentes, sino una perspectiva de comprensión y ejercicio de la actividad científica implicada con la complejidad de lo humano (Morin, 1995). Las limitaciones de los enfoques centrados en la enfermedad y no en la persona, la cada vez mayor prevalencia de problemas crónicos, trastornos mentales y lo que se entiende como multimorbilidad, el auge de la prevención cuaternaria como respuesta a la nociva medicalización de problemas que no la necesitan, y los siempre inseparables vínculos de las personas y poblaciones enfermas con sus contextos biográficos, familiares y sociales están empujando en el seno de la epidemiología y de las corrientes basadas en la evidencia a reconocer la excesiva simplicidad de determinados presupuestos teóricos y la cuestionable efectividad de algunas de sus prácticas (Greenhalgh, 2012; Wilson et al., 2001). También en el campo de la IC deberíamos estar atentos para evitar la simplicidad tanto en los diseños metodológicos como en los desarrollos teóricos relacionados con la práctica investigadora (Barbour \& Barbour, 2003; Pope \& Mays, 2009). complejidad de los fenómenos de salud-enfermedad en las poblaciones y de las respuestas a los mismos desde la atención sanitaria y los servicios de salud constituye por tanto un reto imprescindible para el desarrollo de la IC en este campo.

\section{- El reto de lo inter y transdisciplinario}

A su vez, asumir y responder al reto de la complejidad es una tarea que difícilmente se puede llevar a cabo desde lo monodisciplinario. Para ello es necesaria la colaboración y la interacción entre las diferentes disciplinas e instituciones, tanto sanitarias como no sanitarias, y también la apertura de cauces para la participación de enfermos y ciudadanos (Lambert \& McKevitt, 2002; Park, 2001). Ello requiere por un lado de la humildad consciente señalada por Ortí (Ortí, 1986) en relación al reconocimiento reflexivo de las limitaciones de nuestras lentes habituales. Y por otro de la valentía que supone el desplazamiento nómada hacia las periferias, hacia las zonas de intersección y encuentro con los campos de los otros, con la consiguiente pérdida de seguridad que conlleva relativizar nuestros títulos, nuestros lenguajes y nuestras identidades corporativas. 


\section{- El reto de la defensa de lo público y de la justicia social}

Y finalmente entiendo que no deberíamos ignorar las amenazas presentes en nuestras realidades sociales, económicas y políticas. Investigar y colaborar entre diferentes para intentar dar respuesta a las necesidades de salud de nuestras poblaciones, requiere de apuestas políticas a favor de la consolidación de servicios públicos de calidad tanto en sanidad como en educación que posibiliten la necesaria complementariedad entre asistencia, docencia e investigación en los servicios de salud. Y hoy, tanto en España como en Portugal, y también en otros muchos países de nuestra comunidad iberoamericana, estamos sufriendo con crudeza los efectos de las políticas conservadoras orientadas al derribo de lo público, al aumento de la pobreza y de las desigualdades sociales, y también al incremento del miedo y la insolidaridad entre los ciudadanos. Los recortes presupuestarios en servicios públicos de salud y en investigación son ya una dramática realidad (Ferrer, 2012; Minué-Lorenzo et al., 2012), y el atrincheramiento de cada uno en su parcela de supervivencia constituye, en mi opinión, un grave peligro a evitar si queremos contribuir como investigadores cualitativos a la consecución de sociedades más justas, más libres y más sanas.

\section{BIBLIOGRAFÍA}

BARBOUR, R. S. e M. Barbour (2003), "Evaluating and synthesizing qualitative research: the need to develop a distinctive approach", Journal of Evaluation in Clinical Practice, 9 (2), pp. 179-186.

CALDERÓN, C. (2004), "Las revisiones sistemáticas y el reto de la evaluación de la calidad en la investigación cualitativa en salud", Aten Primaria, 34 (4), pp. 166-169.

CALDERÓN, C. (2009), “Assessing the Quality of Qualitative Health Research: Criteria, Process and Writing”, Forum Qualitative Sozialforschung/Forum: Qualitative Social Research, 10 (2), Art 17-http:// nbn-resolving.de/urn:nbn:de:0114-fqs0902178.

CRESWELL, J. W. (2011), “Controversies in Mixed Methods Research” in N. K. Denzin e Y. S. Lincoln (eds.), The Sage Handbook of Qualitative Research, 4 ed., Thousand Oaks, CA, Sage, pp. 269-283.

FERRER, E. (2012), “Economistas Frente a la Crisis”. Disponível em: http:// economistasfrentealacrisis.wordpress.com/2012/08/29/crisis-economica-salud-y-sistemasanitario/.

GREENHALGH, T. (2012), "Why do we always end up here? Evidence-based medicine's conceptual cul-de-sacs and some off-road alternative routes”, J. Prim. Health Care, 4 (2), pp. 92-97.

LAMBERT, H. e C. McKevitt (2002), “Anthropology in health research: from qualitative methods to multidisciplinarity”, BMJ, 325, pp. 210-213.

MERENSTEIN, J. e G. Rao et al. (2003), “Clinical research in family medicine: quantity and quality of published articles”, Fam. Med., 35 (4), pp. 284-288. 
MINUÉ-LORENZO, S.; J. F. García Gutiérrez et al. (2012), "Beginning of the end for Spain's national health system", BMJ, 344, p. 3213.

MIRA, J. J., V. Pérez-Jover et al. (2004), “La investigación cualitativa: una alternativa también válida”, Aten Primaria, 34 (4), pp. 161-166.

MORIN, E. (1995), Introducción al Pensamiento Complejo, Barcelona, Gedisa.

MORSE, J. M., M. Barrett et al. (2002), "Verification strategies for establishing reliability and validity in qualitative research", IJQM, 1 (2), Article 2-Retrieved from http:// ejournals.library.ualberta.ca/index.php/IJQM/article/view/4603/3756.

NIEDERKROTENTHALER, T., T. E. Dorner et al. (2011), "Development of a practical tool to measure the impact of publications on the society based on focus group discussions with scientists", BMC.Public Health, 11, p. 588.

ORTÍ, A. (1986), "La apertura y el enfoque cualitativo o estructural: la entrevista abierta semidirectiva y la discusión en grupo," in M. García, J. Ibáñez et al (eds.), El análisis de la realidad social. Métodos y técnicas de investigación, Madrid, Alianza Editorial, pp. 153-185.

PALEY, J. e R. Lilford (2011), “Qualitative methods: an alternative view”, in BMJ, 342, p. 424.

PARK, P. (2001), “Knowledge and Participatory Research”, in P. Reason e H. Bradbury, (eds.), Handbook of Action Research. Participative Inquiry \& Practice, Londres, Sage, pp. 81-90.

PAWSON, R.; T. Greenhalgh et al. (2005), "Realist review - a new method of systematic review designed for complex policy interventions", J.Health Serv.Res Policy, 10 Suppl 1, pp. 21-34.

POPE, C. e N. Mays (2009), "Critical reflections on the rise of qualitative research", in BMJ, 339, p. 3425.

PUJOL, E., M. Monteagudo et al. (2011), “Investigación cualitativa en atención primaria de salud: Situación actual, aportaciones y algunos retos", Revista Clínica Electrónica en Atención Primaria, 19 (Editorial) Disponível em: http://www.fbjoseplaporte.org/rceap/articulo2.php? idnum=19\&art=editorial.

REYNOLDS, J.; J. Kizito et al. (2011), "Quality assurance of qualitative research: a review of the discourse”, Health Res. Policy Syst., 9, 43, doi: 10.1186/1478-4505-9-43.

TEDDLIE, C. e A. Tashakkori (2011), "Mixed Methods Research: Contemporary Issues in an Emerging Field", in N. K. Denzin e Y. S. Lincoln, (eds.), The Sage Handbook of Qualitative Research, 4. ed., Thousand Oaks, CA, Sage, pp. 285-299.

WILSON, T.; T. Holt et al. (2001), “Complexity science: complexity and clinical care”, BMJ, 323 (7314), pp. 685-688.

\section{NOTAS}

1. Comunicação apresentada no V Congresso Ibero-Americano de Pesquisa Qualitativa em Saúde - Circulação de Saberes e Desafios em Saúde, realizado em Lisboa entre 11 e 13 de Outubro de 2012. 


\section{RESÚMENES}

La ponencia expone las principales consideraciones del autor a partir de la información extraída del Índice Médico Español sobre las publicaciones cualitativas en salud en España. Se analizan las tendencias y carencias en la evaluación de la producción, los ámbitos de publicación, el origen de los autores, y los enfoques en diseños y contenidos. Finalmente se plantean tres retos desde el presente, relacionados con la importancia de asumir la complejidad en el desarrollo metodológico de la Investigación Cualitativa en los servicios de salud, la necesidad de la colaboración inter y transdisciplinaria, y el imperativo de la defensa de servicios públicos de calidad en la actual crisis económica.

A comunicação expõe as principais considerações do autor a partir da informação extraída do Índice Médico Espanhol sobre as publicações qualitativas em saúde em Espanha. Analisam-se as tendências e as lacunas na avaliação da produção, nas áreas de publicação, na origem dos autores, e nas abordagens em termos de desenhos e conteúdos. Finalmente, identificam-se três desafios atuais, relacionados com a importância de assumir a complexidade no desenvolvimento metodológico da Investigação Qualitativa nos serviços de saúde, a necessidade de colaboração inter e transdisciplinar, e o imperativo da defesa de serviços públicos de qualidade na atual crise económica.

The paper puts forward the author's main considerations about the information extracted from the Spanish Medical Index on qualitative health publications in Spain. The trends and gaps in the assessment of the production, the fields of publishing, the origin of the authors, and the approaches in designs and contents are analyzed. Finally three challenges are discussed, related to the importance of taking into account the complexity in the methodological development of Qualitative Research in the health services, the need for cross-disciplinary collaboration, and the imperative of defending the public services in the current economic crisis.

\section{ÍNDICE}

Keywords: qualitative health research, health services research, literature review

Palavras-chave: investigação qualitativa em saúde, investigação em serviços de saúde, revisão bibliográfica

Palabras claves: investigación cualitativa en salud, investigación en servicios de salud, revisión bibliográfica 